Pier Mauro GIACHINO* - Dante VAILATI**

\title{
Un nuovo Anemadus dell'Italia meridionale (Coleoptera Leiodidae Cholevinae)
}

\begin{abstract}
Riassunto: Gli autori descrivono Anemadus petruzzielloi n. sp. (Coleoptera Leiodidae Cholevinae) delle grotte Strazzatrippa e degli Angeli, Acerno, prov. Salerno (Italia). Vengono forniti i caratteri diagnostici della nuova specie, che appartiene ad un distinto gruppo di specie, e la distribuzione geografica in rapporto alle altre specie note di Anemadus presenti nell'area.
\end{abstract}

\begin{abstract}
A new Anemadus from Southern Italy (Coleoptera, Leiodidae, Cholevinae).
Anemadus petruzzielloi n. sp. (Coleoptera, Leiodidae, Cholevinae) is described from the caves Strazzatrippa and degli Angeli, Acerno, Salerno province (Italy). Diagnostic characters of the new species, belonging to a separate group of species, and its geographical distribution, related to the other known species of Anemadus present in this area, are given.
\end{abstract}

Key words: Coleoptera, Leiodidae, Cholevinae, Anemadini, Anemadus petruzzielloi n. sp., taxonomy, zoogeography.

\section{INTRODUZIONE}

Negli anni immediatamente successivi alla pubblicazione della monografia di Giachino \& Vailati (1993) è sopratutto Perreau (1996a, 1996b, 2000) che si occupa ancora della tribù Anemadini con tre contributi, nei quali discute la posizione sistematica del genere Anemadiola Szymczakowski, 1963 e descrive tre nuove specie di Anemadus di Cina e Giappone. Anche Rủžička (1999) descrive una nuova specie di Anemadus di Cina.

Ad eccezione di una nuova stazione di Anemadus cavazzutii Giachino \& Vailati, 1993, pubblicata nell'ambito di uno studio complessivo sui Cholevinae dell'Anatolia (Giachino \& Vailati, 2000), nessun ulteriore contributo sugli Anemadini del Bacino del Mediterraneo viene pubblicato fino alla fine del secolo scorso.

Negli anni successivi al 2000, mentre Perreau (2002, 2004, 2009) descrive ulteriori cinque nuove specie di Anemadus dell'Asia, sono ancora Giachino \& Vailati (2001) e Giachino et al. (2013) che descrivono tre nuovi Anemadus della Grecia e del Vicino Oriente (Anatolia e Siria): A. whiteheadi Giachino \& Vailati, 2001; A. lucarellii Giachino, Latella, Vailati, 2013; A. kadleci Giachino, Latella, Vailati, 2013. Nello stesso periodo Giachino \& Vailati $(2005,2007)$ e Giachino et al. (2011) pubblicano contributi zoogeografici sui Cholevinae di determinate aree italiane, lavori che includono anche dati faunistici inediti su Anemadus, ma nessuna ulteriore novità tassonomica.

Recentemente, indagini faunistiche compiute da Luigi Petruzziello (Brescia) in alcune grotte della Campania (Italia) hanno evidenziato la presenza di una nuova, inaspettata, specie di Anemadus, la cui descrizione è oggetto della presente nota.

\section{MATERIALI E METODI}

I materiali oggetto del presente contributo provengono tutti dalle ricerche condotte in Campania da Luigi Petruzziello, appassionato entomologo, e giunti in studio ad uno degli autori (PMG) grazie alla cortesia di Arnaldo Bordoni.

I genitali maschili e femminili sono stati dissezionati e, dopo passaggio in $\mathrm{KOH}$ a freddo, disidratati in alcool e chiarificati in xilolo, per essere poi inclusi in Balsamo del Canada e montati, su cartellini di acetato, sotto l'esemplare di riferimento.

I disegni al tratto sono stati realizzati da D.V. tramite un microscopio binoculare Wild M3 con oculare micrometrico per l'habitus e con un microscopio Leitz Dialux equipaggiato con camera lucida per i disegni dei genitali.

\footnotetext{
*Pier Mauro Giachino, Settore Fitosanitario Regionale, Environment Park, Palazzina A2, Via Livorno 60, 10144 Torino, Italia. E-mail: piermauro.giachino@regione.piemonte.it; p.maurogiachino@libero.it

**Dante Vailati, Via Interna 8, 25127 Brescia, Italia. E-mail: dante.vailati@libero.it
} 


\section{Acronimi}

I materiali esaminati sono conservati nelle seguenti collezioni:

CCa Collezione Achille Casale, Torino

CBo: Collezione Arnaldo Bordoni, Firenze

CGi: Collezione Pier Mauro Giachino, Torino

CGr: Collezione Mario Grottolo, Brescia

CPe: Collezione Luigi Petruzziello, Brescia

CVa: Collezione Dante Vailati, Brescia

MCSNG: Museo Civico di Storia Naturale "G. Doria", Genova

Per il materiale tipico sono stati utilizzati i seguenti acronimi:

HT: Holotypus

PT(T): Paratypus (i)

Anemadus petruzzielloi $\mathrm{n}$. sp. (Figg. 1-6)

Località tiPICA. Italia, Campania, Acerno (Sa), Grotta Strazzatrippa, n 598 Cp/SA, 1125 m.

Serie tipica. HT ô, Italia, Campania, Acerno (Sa), Grotta Strazzatrippa, n 598 Cp/SA, 1125 m, 28.XII.2015, L. Petruzziello leg. (CGi).

PTT: 14 万ิ 14 우으, Italia, Campania, Acerno (Sa), Grotta Strazzatrippa, n 598 Cp/SA, 1125 m, 28.XII.2015, L. Petruzziello leg. (MCSNG, CCa, CGi, CPe, CVa); 3 ○ึ 14 우오, Italia, Campania, Acerno (Sa), Grotta Strazzatrippa, n 598 Cp/SA, 1125 m, 31.XII.2016, L. Petruzziello leg. (CGi, CVa); 3 ふ઼ 4 우, Italia, Campania, Acerno (Sa), Grotta Strazzatrippa, n 598 Cp/SA, 1125 m, 1/19.VIII.2014, L. Petruzziello leg. (CBo); 3 đ̋ , Italia, Campania, Acerno (Sa), Grotta Strazzatrippa, n 598 Cp/SA, 1125 m, 27.XII.2014/18.VII.2015, L. Petruzziello leg. (CBo); 2 궈 8 우우, Italia, Campania, Acerno (Sa), Grotta Strazzatrippa, n 598 Cp/SA, 1125 m, 31.XII.2017, L. Petruzziello leg. (CBo); 7 우, Italia, Campania, Acerno (Sa), Grotta degli Angeli, n 588 Cp/SA, 1075 m, XII.2015/23.VII.2016, L. Petruzziello leg. (CGi, CVa); 3 우우, Italia, Campania, Acerno (Sa), Grotta degli Angeli, n 588 Cp/SA, $1100 \mathrm{~m}$, 15.XII.2015/23.VII.2016, L. Petruzziello leg. (CBo); 2 처 2 우, Italia, Campania, Acerno (Sa), Grotta degli Angeli, n 588 Cp/SA, $1075 \mathrm{~m}$, 19.VIII./27.XII.2014, L. Petruzziello leg. (CGr).

DiAGNOSI. Un Anemadus appartenente ad un gruppo di specie distinto rispetto a quelli individuati da Giachino \& Vailati (1993), qui definito come "gruppo di Anema- dus petruzzielloi". Affine ad Anemadus acicularis (Kraatz, 1852) e ad Anemadus italicus (Zoia, 1990) per la struttura generale dei parameri dell'edeago, che presentano un apice regolarmente curvo verso l'interno, non piegato ad uncino verso l'esterno, e per la forma del pronoto, a base ristretta. Differisce tuttavia da queste due specie per la forma dell'apice del lobo mediano dell'edeago ad apice nettamente lanceolato in visione dorsale e per l'assenza delle due fanere dentate basali del sacco interno, tipiche delle specie del "gruppo di A. acicularis" (sensu Giachino \& Vailati, 1993). Proprio per la tipica morfologia dell'apice del lobo mediano dell'edeago, A. petruzzelloi n. sp. potrebbe essere avvicinato ad alcune specie del "gruppo di $A$. strigosus", quali $A$. whiteheadi Giachino \& Vailati, 2001 dell'Isola di Cefalonia (Grecia) o A. kadleci Giachino, Latella, Vailati, 2013 della Siria. Tuttavia si tratta con ogni probabilità di fenomeni di convergenza trattandosi, in questo caso, di taxa appartenenti ad un gruppo di specie molto ben caratterizzato sia come morfologia dell'edeago sia come forma del pronoto, tipicamente a base allargata, non ristretta (Giachino \& Vailati, 1993, 2001; Giachino et al., 2013).

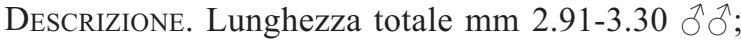
3.04-3.20 우우. Corpo bruno con sfumature rossicce; zampe, antenne e palpi di colore rossiccio scuro; antennomeri 3-11 bruniti.

Tegumenti coperti uniformemente di pubescenza dorato-rossiccia, breve e mediamente eretta.

Capo retrattile, con occhi ben sviluppati; pubescenza breve e coricata sulla fronte, eretta sul clipeo. Antenne moderatamente allungate, gracili, raggiungenti, distese all'indietro, il quarto basale delle elitre. Antennomero $8^{\circ}$ più lungo che largo.

Lunghezza degli antennomeri nell'holotypus (in mm): $0.151-0.138-0.171-0.131-0.171-0.118$ - 0.151- $0.092-0.125-0.131-0.190$

Pronoto trasverso (rapporto larghezza max/lunghezza max: 1.60-1.69 $\widehat{\partial}^{\lambda}$; $1.69-1.82$ 우) con massima larghezza al terzo basale, privo di impressioni basali evidenti; lati regolarmente arcuati, meno in avanti, angoli posteriori ottusi, arrotondati. Base del pronoto larga quanto la base delle elitre, lievemente bisinuata. Pubescenza del disco dorato-rossicia, breve e mediamente eretta. Scultura a granulosità grossolana con microscultura accentuata.

Elitre ellittiche, allungate (rapporto larghezza max/lunghezza max: 0.63-0.66 $\partial^{\lambda} ; 0.58-0.62$ 우 ), 
separatamente arrotondate all'apice, leggermente convergenti nel terzo basale; disco elitrale convesso, spianato lungo la sutura nella metà basale, bruscamente deiscente all'apice. Scultura di tipo "b" (sensu Giachino \& Vailati, 1993). Stria suturale presente, ben marcata, subparallela alla sutura elitrale nei $2 / 3$ basali, bruscamente convergente nel terzo distale. Sono presenti tracce, visibili a luce radente nella parte centrale del disco, delle prime due strie elitrali interne.

Zampe relativamente allungate; protibie poco ispessite all'apice, prive di rigonfiamento latero-ven- trale interno nel maschio, ma, sempre nel maschio, più robuste; meso- e metatibie diritte. Primi tre articoli dei tarsi anteriori dilatati nel maschio, più stretti dell'apice della tibia.

Edeago (Figg. 2, 3) grande, allungato, lungo $\mathrm{mm} 0.93$ (0.96 compresi i parameri). Lobo mediano, in visione dorsale, slanciato, a lati paralleli nella parte basale, che si prolungano convergenti verso la regione apicale; questa è priva di setole, subtriangolare e presenta due evidenti espansioni laterali preapicali e un apice subacuminato. In visione laterale il

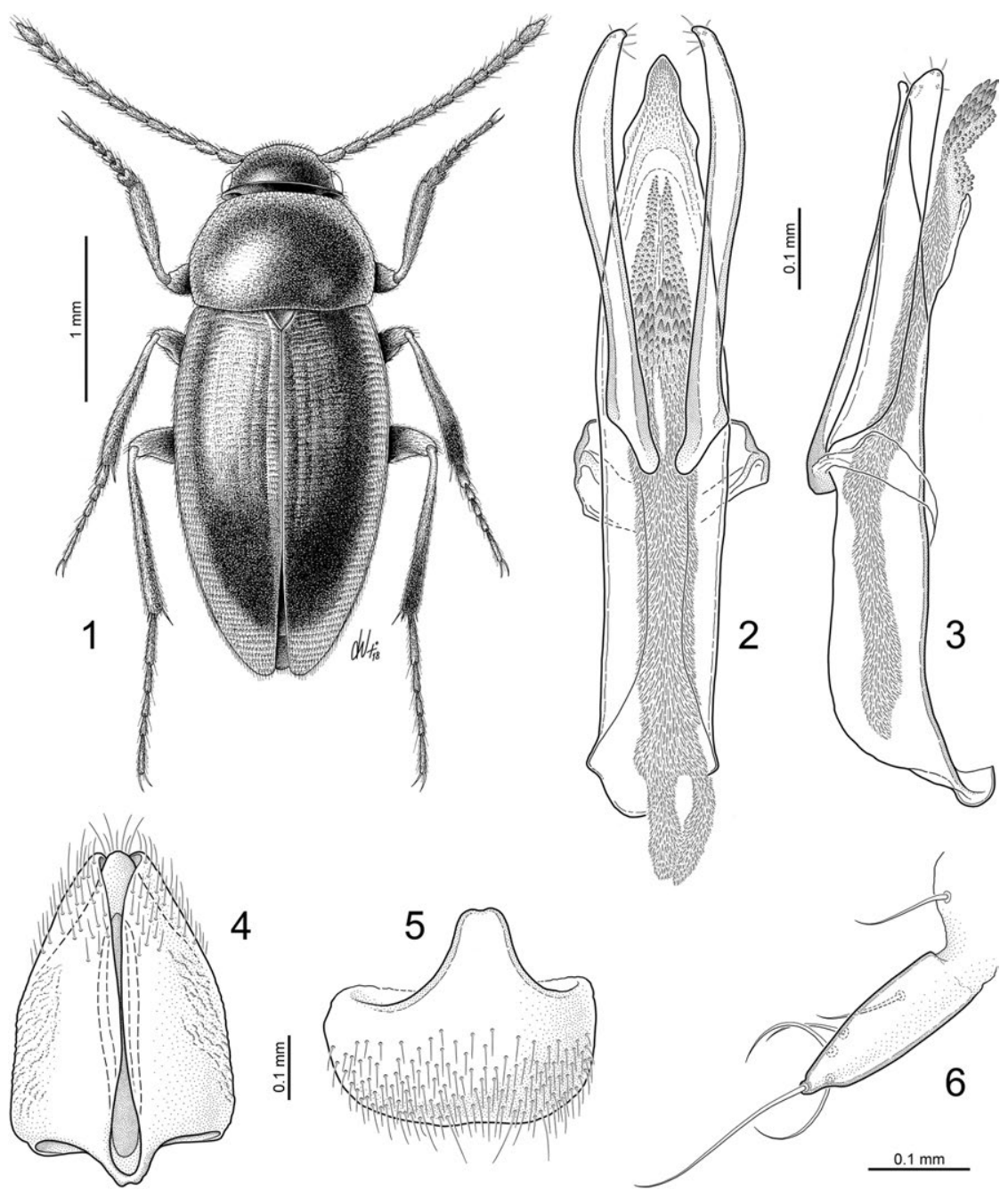

Figg. 1-6. Anemadus petruzzielloi n. sp. 1: habitus (HT §)); 2: lobo mediano in visione dorsale (HT đ); 3: lobo mediano in visione laterale ( $\mathrm{PT} \precsim$ ); 4: segmento genitale maschile in visione ventrale (HT $\precsim$ ); 5: ottavo segmento genitale femminile in visione ventrale (PT $\odot$ ); 6: stilo sinistro in visione dorsale (PT $\odot$ ). 
lobo mediano appare con il profilo dorsale concavo, poi sinuato distalmente, con il tratto apicale subrettilineo.

Parameri (Figg. 2, 3) robusti, spessi dalla base fin quasi all'apice, in visione dorsale leggermente curvati verso l'interno, poi regolarmente assottigliati e con gli apici subdentati, nettamente rivolti all'interno; le setole, in numero di 5, sono inserite all'apice (1) e sul margine interno (4).

Segmento genitale maschile come da Fig. 4.

Sacco interno con fanere sclerificate poco evidenti, munito di due sacche dentate mediane da cui si diparte un grosso fascio spinuloso impari, che si divide in due nella metà basale.

Genitali femminili (Figg. 5, 6) conformi al modello presentato dal genere.

DERIVATIO NOMINIS. Dedichiamo con piacere questa nuova, interessante specie al suo raccoglitore Luigi Petruzziello, appassionato indagatore della fauna sotterranea campana.

DistribuZione ed ECOLOGia. A. petruzzielloi n. sp. è noto al momento di due sole grotte: la Grotta Strazzatrippa (catasto n. $598 \mathrm{Cp} / \mathrm{SA}$ ) e la Grotta degli Angeli (catasto n. $588 \mathrm{Cp} / \mathrm{SA}$ ), dove è stato raccolto con l'utilizzo di trappole lasciate per periodi diversi di tempo.

Le due grotte si aprono entrambe nel comune di Acerno in provincia di Salerno, fanno parte di uno stesso complesso carsico e sono comunicanti fra loro (Bordoni, 2018).

Fra la fauna associata, già parzialmente citata da Bordoni (2018), sono presenti, fra i Leiodidae Cholevinae: Choleva sturmi Brisout, 1863, C. angustata (Fabricius, 1781), C. reitteri Petri, 1915, Catops subfuscus Kellner, 1846, C. tristis (Panzer, 1793) e Bathysciola partenii Ruffo,1947. Di particolare importanza la presenza di Lathrobium petruzzielloi Bordoni, 2018 (Staphylinidae), elemento molto specializzato alla vita in ambiente sotterraneo.

OsSERVAZIONI. La scoperta, nell'Appennino meridionale, di una nuova specie di Anemadus è del tutto inaspettata. Nella stessa area geografica erano note, al momento, altre tre specie di Anemadus pertinenti a due diversi gruppi di specie: A. acicularis (Kraatz, 1852) e A. italicus (Zoia, 1990) appartenenti al "gruppo di A. acicularis" (sensu Giachino \& Vailati, 1993) e A. strigosus strigosus (Kraatz, 1852) appartenente al "gruppo di A. strigosus" (sensu Giachino \& Vailati, 1993). Leggermente più a settentrione, nel Lazio, occorre ricordare anche la presenza di una seconda specie di Anemadus del "gruppo di A. strigosus": A. karamani Ganglbauer, 1899 (Fig. 7), mentre in Sicilia è presente A. osellai Giachino \& Vailati, 1993, del "gruppo di A. acicularis".

Come già evidenziato nella diagnosi, $A$. petruzzielloi $\mathrm{n}$. sp., pur presentando una struttura del lobo mediano dell'edeago riconducibile al modello che caratterizza le specie del "gruppo di A. acicularis" (parameri tozzi, convergenti apicalmente e privi di espansioni dentiformi rivolte esternamente), si diffe-

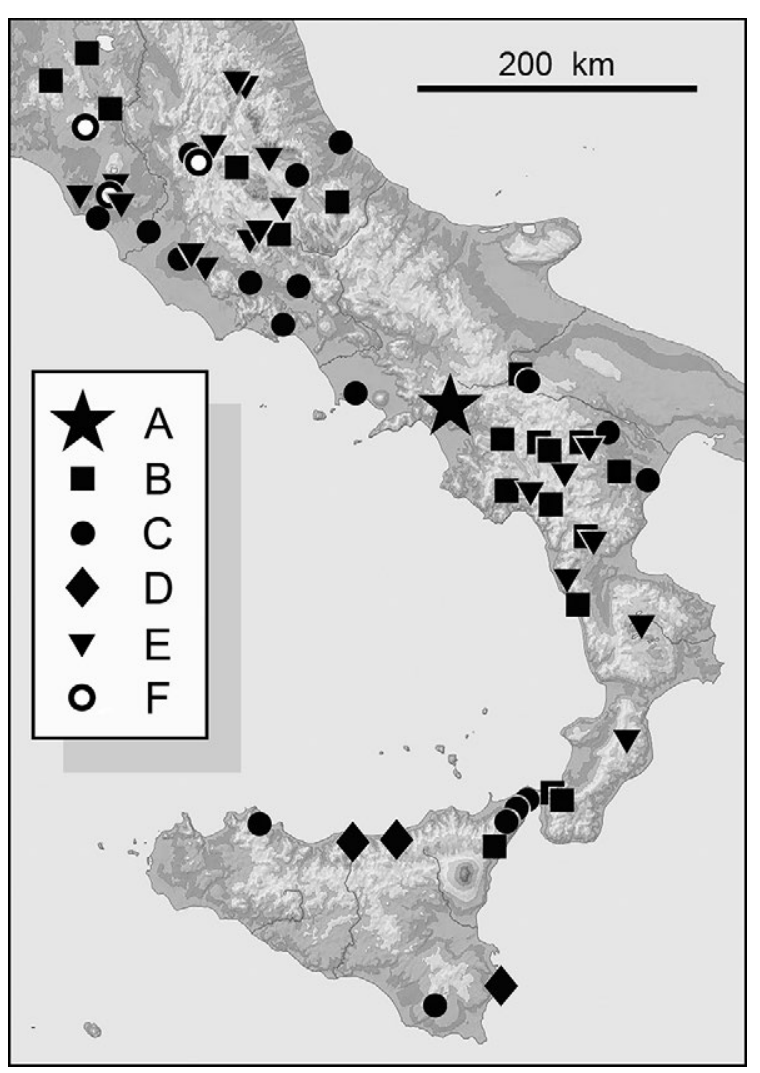

Fig. 7. Distribuzione nota delle specie di Anemadus nell'Appennino centro meridionale e in Sicilia. A: A. petruzzielloi n. sp.; B: A. italicus (Zoia, 1990); C: A. acicularis (Kraatz, 1852); D: A. osellai Giachino \& Vailati, 1993; E: A. strigosus strigosus (Kraatz, 1852); F: A. karamani Ganglbauer, 1899. 
renzia da tutte le specie di questo gruppo per l'assenza delle fanere dentate basali dell'endofallo (Figg. 2-3). Per questo motivo la specie viene considerata pertinente ad un gruppo di specie distinto, "gruppo di $A$. petruzzielloi" (sensu novo), affine al "gruppo di A. acicularis" (sensu Giachino \& Vailati, 1993).

\section{RINGRAZIAMENTI}

Siamo molto grati a Luigi Petruzziello e ad Arnaldo Bordoni per averci concesso in studio il materiale in loro possesso.

Un ringraziamento particolare ad Achille Casale per la revisione del manoscritto.

\section{BIBLIOGRAFIA}

Bordoni A., 2018 - A new species of glyptomeroid Lathrobium from Campania, Italy (Coleoptera, Staphylinidae). Bollettino della Società entomologica italiana, 130 (1): 41-46.

Giachino P.M., VAILATI D., 1993 - Revisione degli Anemadinae. Monografie di Natura Bresciana, 18, 314 pp.

Giachino P.M., VAILATI D., 2000 - I Cholevidae del Vicino Oriente: inventario, analisi faunistica e origine del popolamento (Coleoptera). Biogeographia, 21: 7-172.

Giachino P.M., Vallati D., 2001 - Nuovi dati sugli Anemadinae con descrizione di Anemadus whiteheadi sp. n. (Coleoptera: Cholevidae). Elytron, 14 (2000), 119-124.

Giachino P. M., VAILATI D., 2005 - I Cholevidae delle Alpi e Prealpi italiane: inventario, analisi faunistica e origine del popolamento nel settore compreso fra i corsi dei fiumi Ticino e Tagliamento. Biogeographia, 26: 229-377.

Giachino P. M., VAILATI D., 2007 - I Coleotteri Colevidi dell'Appennino settentrionale e centrale: inventario, analisi faunistica e origine del popolamento (Coleoptera Cholevidae). Biogeographia, 27: 365-420.

Giachino P.M., Latella L., Vailati D., 2013 - Two new species of the genus Anemadus Reitter, 1885, from the Near East (Coleoptera: Cholevidae). Zootaxa, 3718(4): 378-386.

Giachino P. M., VAilati D., BAVIERA C., 2011 - I Coleotteri Colevidi della Sicilia: inventario, analisi faunistica e origine del popolamento (Coleoptera, Cholevidae). Biogeographia, 30: 467- 484.

JeAnnel R., 1936 - Monographie des Catopidae. Mémoires du Muséum national d'Histoire naturelle Paris, (n.s.), 1, 1-433

Perreau M., 1996a - Contribution à la connaissance des Cholevidae du Japon et de Taiwan (Coleoptera). Revue Suisse de Zoologie, 103(1): 283-297.

Perreau M., 1996b - Nouveaux Cholevinae d'Asie (Coleoptera Leiodidae). Revue Suisse de Zoologie, 103(4): 939-949.

PerReAu M., 2000 - Catalogue des Coléoptères Leiodidae Cholevinae et Platypsyllinae. Mémoires de la Société entomologique de France, 4, 460 pp.

PerReAu M., 2002 - Nouvelles espèces de Leiodidae Cholevinae (Coleoptera), notes sur quelques espèces mal connues et correction d'une homonymie. Mitteilungen der Schweizerischen Entomologischen Gesellschaft, 75: 41-50.

Perreau M., 2004 - Contribution à la connaissance des Leiodidae (Coleoptera). Mitteilungen der Schweizerischen Entomologischen Gesellschaft, 77: 197-212.

Perreau M., 2009 - Nouveaux Ptomaphagini et Anemadini souterrains, dont la première espèce anophtalme d'Anemadus (Coleoptera: Leiodidae Cholevinae). Annales de la Société entomologique de France, 45: 1-10.

RŮŽIČKA J., 1999 - A new apterous and microphthalmic species of Anemadus (Coleoptera: Leiodidae: Cholevinae) from China. Revue Suisse de Zoologie, 106(3): 621-626. 